\title{
Leptonic Probes of the Standard Model
}

\author{
A. Pich ${ }^{\mathrm{a}}$ \\ a Departament de Física Teòrica, IFIC, Universitat de València - CSIC, \\ Apt. Correus 22085, E-46071 València, Spain
}

Precise measurements of the lepton properties provide stringent tests of the Standard Model structure and accurate determinations of its parameters. We overview the present status of a few selected topics: lepton universality, QCD tests and the determination of $\alpha_{s}$ and $m_{s}$ from hadronic $\tau$ decays, the anomalous lepton magnetic moments and neutrino oscillations.

\section{INTRODUCTION}

The Standard Model constitutes one of the most successful achievements in modern physics. It is a very elegant theoretical framework, which is able to describe nearly all known experimental facts in particle physics [1,2].

The known leptons have provided clean probes to perform very precise tests of the electroweak gauge structure, at the $0.1 \%$ to $1 \%$ level. Moreover, the hadronic $\tau$ decays turn out to be a beautiful laboratory for studying strong interaction effects at low energies 3 3, $1,5,6$. 6 . Accurate determinations of the QCD coupling and the strange quark mass have been obtained with $\tau$ decay data.

Very recently, the first hints of new physics beyond the Standard Model have also emerged from the lepton sector. Convincing evidence of neutrino oscillations has been obtained by SNO (7) and Super-Kamiokande [8,9]. Combined with data from other neutrino experiments, it shows that $\nu_{e} \rightarrow \nu_{\mu, \tau}$ and $\nu_{\mu} \rightarrow \nu_{\tau}$ lepton-flavourviolating transitions do occur [10].

\section{LEPTON UNIVERSALITY}

In the Standard Model all lepton doublets have identical couplings to the $W$ boson:

$\mathcal{L}=\frac{g}{2 \sqrt{2}} W_{\mu}^{\dagger} \sum_{l} \bar{\nu}_{l} \gamma^{\mu}\left(1-\gamma_{5}\right) l+$ h.c.

Comparing the measured decay widths of leptonic or semileptonic decays which only differ by
Table 1

Present constraints on $\left|g_{l} / g_{l^{\prime}}\right|$.

\begin{tabular}{lc}
\hline & $\left|g_{\mu} / g_{e}\right|$ \\
\hline$B_{\tau \rightarrow \mu} / B_{\tau \rightarrow e}$ & $0.9999 \pm 0.0020$ \\
$B_{\pi \rightarrow e} / B_{\pi \rightarrow \mu}$ & $1.0017 \pm 0.0015$ \\
$B_{W \rightarrow \mu} / B_{W \rightarrow e}$ & $1.000 \pm 0.011$ \\
\hline \hline & $\left|g_{\tau} / g_{\mu}\right|$ \\
\hline$B_{\tau \rightarrow e} \tau_{\mu} / \tau_{\tau}$ & $1.0004 \pm 0.0023$ \\
$\Gamma_{\tau \rightarrow \pi} / \Gamma_{\pi \rightarrow \mu}$ & $0.9999 \pm 0.0036$ \\
$\Gamma_{\tau \rightarrow K} / \Gamma_{K \rightarrow \mu}$ & $0.979 \pm 0.017$ \\
$B_{W \rightarrow \tau} / B_{W \rightarrow \mu}$ & $1.026 \pm 0.014$ \\
\hline \hline & $\left|g_{\tau} / g_{e}\right|$ \\
\hline$B_{\tau \rightarrow \mu} \tau_{\mu} / \tau_{\tau}$ & $1.0002 \pm 0.0022$ \\
$B_{W \rightarrow \tau} / B_{W \rightarrow e}$ & $1.026 \pm 0.014$ \\
\hline
\end{tabular}

the lepton flavour, one can test experimentally that the $W$ interaction is indeed the same, i.e. that $g_{e}=g_{\mu}=g_{\tau} \equiv g$. As shown in Table 1, the present data [11,12,13 verify the universality of the leptonic charged-current couplings to the $0.2 \%$ level.

The values of $B_{\tau \rightarrow \mu / e}$ and $\tau_{\tau}$ are already known with a precision of $0.29 \%$ and $0.34 \%$, respectively [13]. It remains to be seen whether BABAR and BELLE could make further improvements. The $\mu$ lifetime has been measured to a much better precision of $10^{-5}$. The universality tests require also 


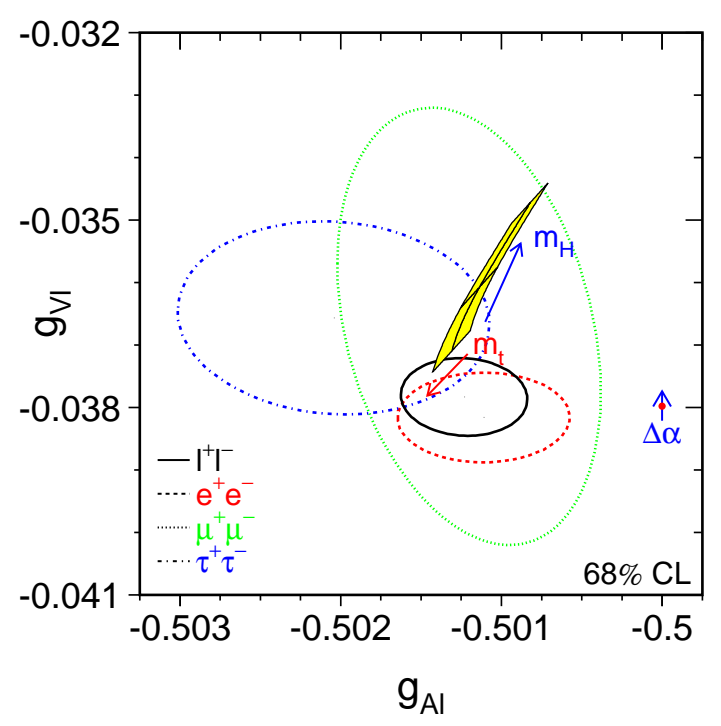

Figure 1. $68 \%$ probability contours in the $a_{l}-v_{l}$ plane from LEP and SLD data [12]. The shaded region is the Standard Model prediction for $m_{t}=$ $174.3 \pm 5.1 \mathrm{GeV}$ and $m_{H}=300_{-186}^{+700} \mathrm{GeV}$.

a good determination of $m_{\tau}^{5}$, which is only known to the $0.08 \%$ level. An improved measurement of the $\tau$ mass could be expected from CLEO-C 14] and CMD-2 [15], through a detailed analysis of $\sigma\left(e^{+} e^{-} \rightarrow \tau^{+} \tau^{-}\right)$at threshold [16].

The interactions of the neutral $Z$ boson are diagonal in flavour. Moreover, all fermions with equal electric charge have identical vector, $v_{f}=$ $T_{3}^{f}\left(1-4\left|Q_{f}\right| \sin ^{2} \theta_{W}\right)$, and axial-vector, $a_{f}=$ $T_{3}^{f}= \pm 1 / 2$, couplings to the $Z$. This has been accurately tested at LEP and SLD through a precise analysis of $e^{+} e^{-} \rightarrow \gamma, Z \rightarrow f \bar{f}$ data. Figure 1 shows the $68 \%$ probability contours in the $a_{l}-v_{l}$ plane, obtained from leptonic observables 12]. The universality of the leptonic $Z$ couplings is now verified to the $0.15 \%$ level for $a_{l}$, while only a few per cent precision has been achieved for $v_{l}$ due to the smallness of the leptonic vector coupling. Assuming universality, the measured leptonic asymmetries provide an accurate determination of the electroweak mixing angle [12]:

$\sin ^{2} \theta_{W}=0.23113 \pm 0.00021$.

\section{HADRONIC TAU DECAYS}

The semileptonic decay modes $\tau^{-} \rightarrow \nu_{\tau} H^{-}$ probe the matrix element of the left-handed charged current between the vacuum and the final hadronic state $H^{-}$.

For the decay modes with lowest multiplicity, $\tau^{-} \rightarrow \nu_{\tau} \pi^{-}$, and $\tau^{-} \rightarrow \nu_{\tau} K^{-}$, the relevant matrix elements are already known from the measured decays $\pi^{-} \rightarrow \mu^{-} \bar{\nu}_{\mu}$ and $K^{-} \rightarrow \mu^{-} \bar{\nu}_{\mu}$. The corresponding $\tau$ decay widths can then be accurately predicted. As shown in Table 1, these predictions are in good agreement with the measured values, and provide a quite precise test of charged-current universality.

For the two-pion final state, the hadronic matrix element is parameterized in terms of the socalled pion form factor $\left[s \equiv\left(p_{\pi^{-}}+p_{\pi^{0}}\right)^{2}\right]$ :

$\left\langle\pi^{-} \pi^{0}\left|\bar{d} \gamma^{\mu} u\right| 0\right\rangle \equiv \sqrt{2} F_{\pi}(s)\left(p_{\pi^{-}}-p_{\pi^{0}}\right)^{\mu}$.

A dynamical understanding of the pion form factor can be achieved 17, 18, 19, 20, by using analyticity, unitarity and some general properties of QCD.

At low momentum transfer, the coupling of any number of $\pi$ 's, $K$ 's and $\eta$ 's to the $\mathrm{V}-\mathrm{A}$ current can be rigorously calculated with Chiral Perturbation Theory [21,22] techniques, as an expansion in powers of $s$ and light quark masses over the chiral symmetry breaking scale $\left(\Lambda_{\chi} \sim 1 \mathrm{GeV}\right)$. This includes chiral loop corrections, which encode the absorptive contributions required by unitarity. The short-distance information is contained in the so-called chiral couplings, which are known to be dominated by the effect of the lowest-mass resonances 23.

In the limit of an infinite number of quark colours $N_{C}$, QCD reduces to a theory of treelevel resonance exchanges 24, 25]. Thus, the $\rho$ propagator governs the pion form factor at $\sqrt{s} \lesssim 1 \mathrm{GeV}$, providing an all-order resummation of the polynomial chiral corrections. The relevant $\rho$ couplings are determined requiring $F_{\pi}(s)$ to satisfy the correct QCD behaviour at large $s$ [23]. The leading $1 / N_{C}$ corrections correspond to pion loops and can be incorporated by matching the large $-N_{C}$ result with the Chiral Perturbation Theory description [17]. Using analyticity and 


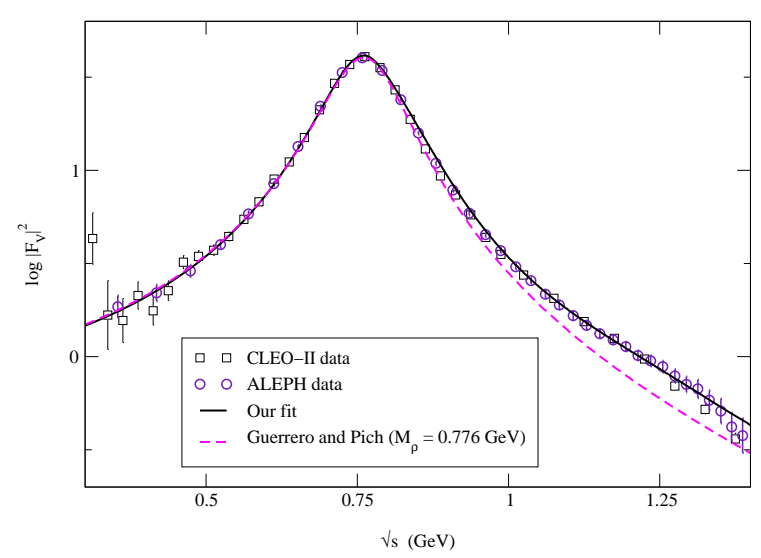

Figure 2. Pion form factor data [26, 27] compared with theoretical predictions 19 .

unitarity constraints, the chiral logarithms associated with those pion loops can be exponentiated to all orders in the chiral expansion. Putting all these fundamental ingredients together, one gets the result [17]:

$$
F_{\pi}(s)=\frac{M_{\rho}^{2}}{M_{\rho}^{2}-s-i M_{\rho} \Gamma_{\rho}(s)} \exp \left\{-\frac{s \operatorname{Re} A(s)}{96 \pi^{2} f_{\pi}^{2}}\right\},
$$

where

$A(s) \equiv \log \left(\frac{m_{\pi}^{2}}{M_{\rho}^{2}}\right)+8 \frac{m_{\pi}^{2}}{s}-\frac{5}{3}+\sigma_{\pi}^{3} \log \left(\frac{\sigma_{\pi}+1}{\sigma_{\pi}-1}\right)$

contains the one-loop chiral logarithms, $\sigma_{\pi} \equiv$ $\sqrt{1-4 m_{\pi}^{2} / s}$ and the off-shell $\rho$ width 17,18 is given by

$\Gamma_{\rho}(s)=\theta\left(s-4 m_{\pi}^{2}\right) \sigma_{\pi}^{3} M_{\rho} s /\left(96 \pi f_{\pi}^{2}\right)$.

This prediction, which only depends on $M_{\rho}, m_{\pi}$ and the pion decay constant $f_{\pi}$, is compared with the data in Fig. 2. The agreement is rather impressive and extends to negative $s$ values, where the $e^{-} \pi$ elastic data (not shown in the figure) sits.

The small effect of higher $\rho$ resonance contributions and additional next-to-leading in $1 / N_{C}$ corrections can be easily included, at the price of having some free parameters which decrease the predictive power 19,20]. This gives a better description of the $\rho^{\prime}$ shoulder around $1.2 \mathrm{GeV}$.
The dynamical structure of other hadronic final states can be investigated in a similar way. The more involved $\tau \rightarrow \nu_{\tau} 4 \pi$ and $e^{+} e^{-} \rightarrow 4 \pi$ transitions have been already studied recently 228 . A theoretical analysis of the $\tau \rightarrow \nu_{\tau} 3 \pi$ data [29, 30 is in progress [31].

\section{LEPTON ANOMALOUS MAGNETIC MOMENTS}

The most stringent QED test [32,33,34 comes from the high-precision measurements of the $e$ [35] and $\mu$ [36] anomalous magnetic moments $a_{l} \equiv\left(g_{l}^{\gamma}-2\right) / 2$ :

$a_{e}=(115965218.69 \pm 0.41) \cdot 10^{-11}$,

$a_{\mu}=(116592030 \pm 80) \cdot 10^{-11}$.

To a measurable level, $a_{e}$ arises entirely from virtual electrons and photons; these contributions are known [32,33] to $O\left(\alpha^{4}\right)$. The impressive agreement achieved between theory and experiment has promoted QED to the level of the best theory ever built to describe nature. The theoretical error is dominated by the uncertainty in the input value of the QED coupling $\alpha$. Turning things around, $a_{e}$ provides the most precise determination of the fine structure constant:

$\alpha^{-1}=137.03599876 \pm 0.00000052$.

The central value is slightly smaller (by 0.82 . $10^{-6}$ ) than the usually quoted value [32], due to a small error recently discovered in the $O\left(\alpha^{4}\right)$ contribution to $a_{l}$ 33].

The anomalous magnetic moment of the muon is sensitive to small corrections from virtual heavier states; compared to $a_{e}$, they scale as $m_{\mu}^{2} / m_{e}^{2}$. The Standard Model prediction can be decomposed in five types of contributions:

$$
\begin{array}{rlll}
10^{11} \cdot a_{\mu}^{\text {th }}=116 & 584718 \pm & \pm & \text { QED [32, 33, 34] } \\
+ & 152 \pm 4 & \text { EW [34, 37] } \\
+6943 \pm 85 & \text { had, LO [38] } \\
-\quad 100 \pm 6 & \text { had, NLO [39] } \\
+\quad 90 \pm 40 & \text { lbl 44, 41] } \\
=116591803 \pm 94 & &
\end{array}
$$

This result differs by $1.8 \sigma$ from the experimental value (6). 


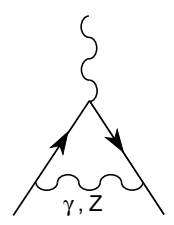

(a)

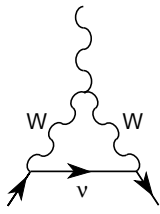

(b)

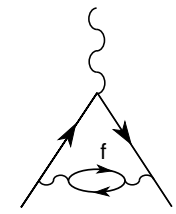

(c)

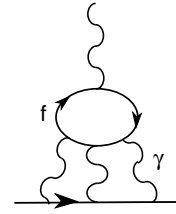

(d)
Figure 3. Feynman diagrams contributing to $a_{l}$.

The main theoretical uncertainty on $a_{\mu}$ has a QCD origin. Since quarks have electric charge, virtual quark-antiquark pairs induce hadronic vacuum polarization corrections to the photon propagator (Fig. 3.c). Owing to the nonperturbative character of QCD at low energies, the light-quark contribution cannot be reliably calculated at present. Fortunately, this effect can be extracted from the measurement of the cross-section $\sigma\left(e^{+} e^{-} \rightarrow\right.$ hadrons) and from the invariant-mass distribution of the final hadrons in $\tau$ decays 19,42 . The largest contribution comes from the $2 \pi$ final state. The $\tau$ decay determination includes a careful investigation of isospin breaking effects 43], using the pion form factor expression of ref. [17], which amount to 33] $\Delta a_{\mu}^{\mathrm{had}}=-(112 \pm 28) \cdot 10^{-11}$.

At present, there is a discrepancy between the $2 \pi$ contributions extracted from $e^{+} e^{-}$and $\tau$ data, which translates into slightly different predictions $(1.8 \sigma)$ for $a_{\mu}$. One gets 38] $a_{\mu}^{\text {had,LO }}=(6847 \pm$ 70) $\cdot 10^{-11}$ from $e^{+} e^{-}$, while the $\tau$ data gives $a_{\mu}^{\text {had,LO }}=(7019 \pm 62) \cdot 10^{-11}$, in better agreement with the BNL-821 measurement of $a_{\mu}$ |36|. In order to quote a reference number for $a_{\mu}^{\text {th }}$, I have used a weighted average of these two determinations, increasing the error with the appropriate scale factor 11. New precise $e^{+} e^{-}$and $\tau$ data sets are needed to settle the true value of $a_{\mu}^{\text {had,LO }}$ [38,44].

Additional QCD uncertainties stem from the smaller light-by-light scattering contributions (Fig. 3.d). A recent reevaluation of these corrections [40] has detected a sign mistake in previous calculations 41], improving the agreement with the experimental measurement [36].
The Brookhaven $(g-2)$ experiment [36] is expected to push its sensitivity to at least $4 \cdot 10^{-10}$, and thereby observe the contributions from virtual $W^{ \pm}$and $Z$ bosons [34,37. A meaningful test of the electroweak Standard Model contributions would require a better control of the QCD corrections.

\section{THE TAU HADRONIC WIDTH}

The inclusive character of the total $\tau$ hadronic width renders possible an accurate calculation of the ratio 45, 46, 47, 48, 49.

$R_{\tau} \equiv \frac{\Gamma\left[\tau^{-} \rightarrow \nu_{\tau} \text { hadrons }(\gamma)\right]}{\Gamma\left[\tau^{-} \rightarrow \nu_{\tau} e^{-} \bar{\nu}_{e}(\gamma)\right]}$,

using analyticity constraints and the Operator Product Expansion. One can separately compute the contributions associated with specific quark currents:

$R_{\tau}=R_{\tau, V}+R_{\tau, A}+R_{\tau, S}$.

$R_{\tau, V}$ and $R_{\tau, A}$ correspond to the Cabibboallowed decays through the vector and axialvector currents, while $R_{\tau, S}$ contains the remaining Cabibbo-suppressed contributions.

The theoretical prediction for $R_{\tau, V+A}$ can be expressed as 47.

$R_{\tau, V+A}=N_{C}\left|V_{u d}\right|^{2} S_{\mathrm{EW}}\left\{1+\delta_{\mathrm{EW}}^{\prime}+\delta_{\mathrm{P}}+\delta_{\mathrm{NP}}\right\}$,

with $N_{C}=3$. The factors $S_{\mathrm{EW}}=1.0194$ and $\delta_{\mathrm{EW}}^{\prime}=0.0010$ contain the electroweak corrections at the leading [50] and next-to-leading [51] logarithm approximation. The dominant correction $(\sim 20 \%)$ is the purely perturbative contribution $\delta_{\mathrm{P}}$, which is fully known to $O\left(\alpha_{s}^{3}\right)$ 47 and includes a resummation of the most important higher-order corrections [48].

Non-perturbative contributions are suppressed by six powers of the $\tau$ mass 47 and, therefore, are very small. Their numerical size has been determined from the invariant-mass distribution of the final hadrons in $\tau$ decay, through the study of weighted integrals [52],

$R_{\tau}^{k l} \equiv \int_{0}^{m_{\tau}^{2}} d s\left(1-\frac{s}{m_{\tau}^{2}}\right)^{k}\left(\frac{s}{m_{\tau}^{2}}\right)^{l} \frac{d R_{\tau}}{d s}$, 


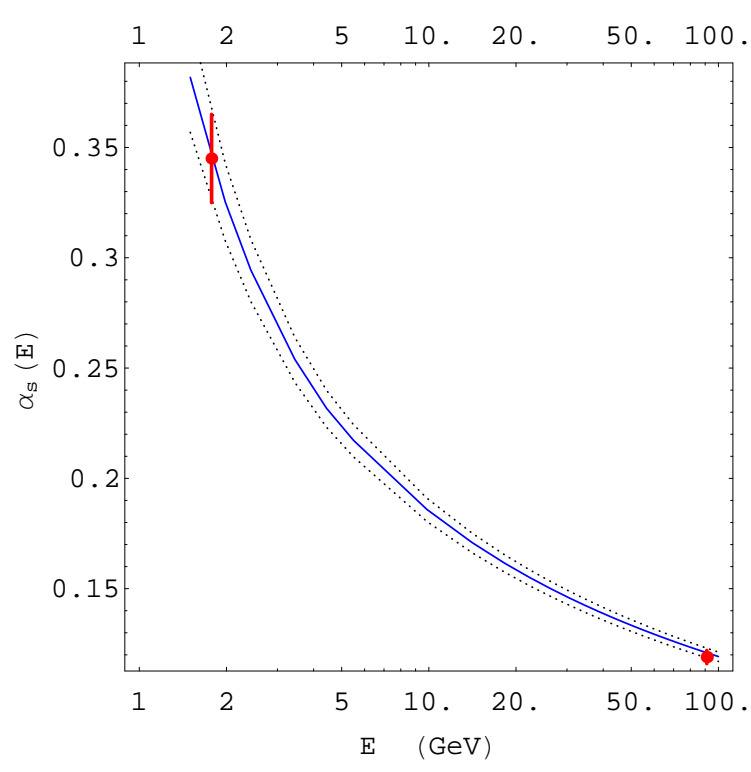

Figure 4. Measured values of $\alpha_{s}$ in $\tau$ and $Z$ decays. The curves show the energy dependence predicted by QCD, using $\alpha_{s}\left(m_{\tau}^{2}\right)$ as input.

which can be calculated theoretically in the same way as $R_{\tau}$. The predicted suppression [47] of the non-perturbative corrections has been confirmed by ALEPH [53], CLEO [54] and OPAL [55]. The most recent analyses [53.55] give

$\delta_{\mathrm{NP}}=-0.003 \pm 0.003$.

The QCD prediction for $R_{\tau, V+A}$ is then completely dominated by the perturbative contribution; non-perturbative effects being smaller than the perturbative uncertainties from uncalculated higher-order corrections. The result turns out to be very sensitive to the value of $\alpha_{s}\left(m_{\tau}^{2}\right)$, allowing for an accurate determination of the fundamental QCD coupling. The experimental measurement 53 55 $R_{\tau, V+A}=3.484 \pm 0.024$ implies $\delta_{\mathrm{P}}=0.200 \pm 0.013$, which corresponds (in the $\overline{\mathrm{MS}}$ scheme) to

$\alpha_{s}\left(m_{\tau}^{2}\right)=0.345 \pm 0.020$.

The strong coupling measured at the $\tau$ mass scale is significantly larger than the values ob- tained at higher energies. From the hadronic decays of the $Z$, one gets $\alpha_{s}\left(M_{Z}^{2}\right)=0.119 \pm 0.003$ 12], which differs from the $\tau$ decay measurement by eleven standard deviations. After evolution up to the scale $M_{Z}$ [56], the strong coupling constant in (12) decreases to

$\alpha_{s}\left(M_{Z}^{2}\right)=0.1208 \pm 0.0025$,

in excellent agreement with the direct measurements at the $Z$ peak and with a similar accuracy. The comparison of these two determinations of $\alpha_{s}$ in two extreme energy regimes, $m_{\tau}$ and $M_{Z}$, provides a beautiful test of the predicted running of the QCD coupling; i.e. a very significant experimental verification of asymptotic freedom.

\section{THE STRANGE QUARK MASS}

The LEP experiments and CLEO have performed an extensive investigation of kaon production in $\tau$ decays [5, 11. ALEPH has determined the inclusive invariant mass distribution of the final hadrons in the Cabibbo-suppressed decays 577. A similar analysis from OPAL should appear soon 58. The separate measurement of the $|\Delta S|=0$ and $|\Delta S|=1$ decay widths allows us to pin down the $\mathrm{SU}(3)$ breaking effect induced by the strange quark mass, through the differences:

$$
\begin{aligned}
\delta R_{\tau}^{k l} & \equiv \frac{R_{\tau, V+A}^{k l}}{\left|V_{u d}\right|^{2}}-\frac{R_{\tau, S}^{k l}}{\left|V_{u s}\right|^{2}} \\
& \approx 24 \frac{m_{s}^{2}\left(m_{\tau}^{2}\right)}{m_{\tau}^{2}} \Delta_{k l}\left(\alpha_{s}\right)-48 \pi^{2} \frac{\delta O_{4}}{m_{\tau}^{4}} Q_{k l}\left(\alpha_{s}\right) .
\end{aligned}
$$

The perturbative QCD corrections $\Delta_{k l}\left(\alpha_{s}\right)$ and $Q_{k l}\left(\alpha_{s}\right)$ are known [59] to $O\left(\alpha_{s}^{3}\right)$ and $O\left(\alpha_{s}^{2}\right)$, respectively. The small non-perturbative contribution, $\delta O_{4} \equiv\left\langle 0\left|m_{s} \bar{s} s-m_{d} \bar{d} d\right| 0\right\rangle=-(1.5 \pm$ $0.4) \times 10^{-3} \mathrm{GeV}^{4}$, has been estimated with Chiral Perturbation Theory techniques [59]. Table 2 shows the measured differences $\delta R_{\tau}^{k l}$ [57,60 and the corresponding $(\overline{\mathrm{MS}})$ values [60] of $m_{s}\left(m_{\tau}^{2}\right)$. The theoretical errors are dominated by the very large perturbative uncertainties of $\Delta_{k l}\left(\alpha_{s}\right)$ 59, 61, 62, 63, 64.

A global analysis, using the information from the three moments and taking into account the strong error correlations, gives the result [60]

$$
m_{s}\left(m_{\tau}^{2}\right)=\left(120 \pm 11_{\mathrm{exp}} \pm 8_{V_{u s}} \pm 19_{\mathrm{th}}\right) \mathrm{MeV} .
$$




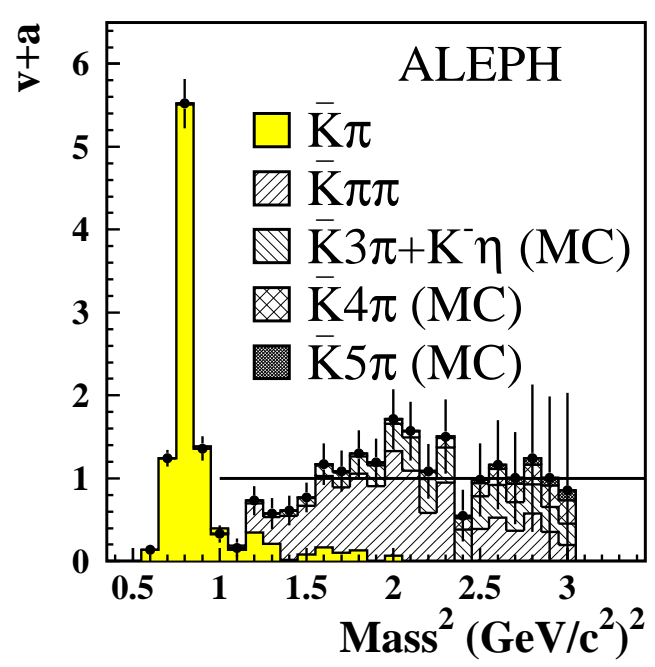

Figure 5. $|\Delta S|=1$ spectral function 57.

Table 2

Measured moments $\delta R_{\tau}^{k l}$ [57,60 and corresponding $m_{s}\left(m_{\tau}^{2}\right)$ values [60].

\begin{tabular}{c|c|c}
$(k, l)$ & $\delta R_{\tau}^{k l}$ & $m_{s}\left(m_{\tau}^{2}\right)(\mathrm{MeV})$ \\
\hline$(0,0)$ & $0.374 \pm 0.133$ & $132 \pm 29_{\exp } \pm 14_{\mathrm{th}}$ \\
$(1,0)$ & $0.398 \pm 0.078$ & $120 \pm 16_{\exp } \pm 16_{\mathrm{th}}$ \\
$(2,0)$ & $0.399 \pm 0.054$ & $117 \pm 12_{\mathrm{exp}} \pm 21_{\mathrm{th}}$
\end{tabular}

This corresponds to $m_{s}\left(\mu^{2}\right)=\left(116_{-25}^{+20}\right) \mathrm{MeV}$ at $\mu=2 \mathrm{GeV}$. A similar result is obtained from an analysis based on "optimal moments" with improved perturbative convergence [65].

Figure 6 compares the $m_{s}$ value obtained from $\tau$ decays with other recent estimates, at the reference scale $\mu=2 \mathrm{GeV}$. There is a rather large spread of lattice results, but the quoted average [66] agrees with the $\tau$ determination. The latest QCD sum rules results [67,68] are also in nice agreement with the $\tau$ value. The advantage of the $\tau$ decay width is the direct use of experimental input, which makes easier to quantify the associated uncertainties. It suffers, however, from a large theoretical uncertainty associated with the bad perturbative behaviour of the scalar contributions to $\Delta_{k l}\left(\alpha_{s}\right)[59,61,62,63,64$. This could

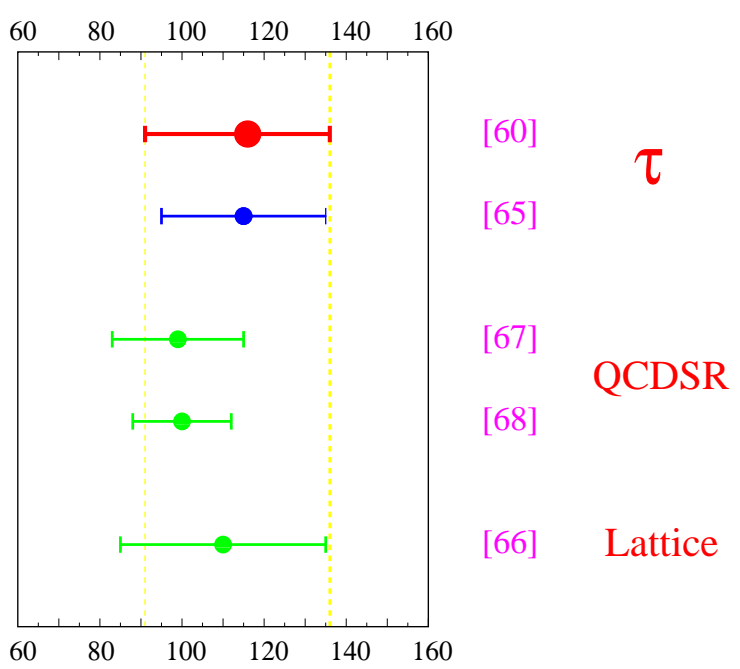

Figure 6. $m_{s}\left(\mu^{2}\right)$ determinations at $\mu=2 \mathrm{GeV}$.

be improved subtracting theoretically the $J=0$ piece, with the help of $K \pi$ scattering data [69].

The strange quark mass is an important input to predict the CP-violating ratio $\varepsilon_{K}^{\prime} / \varepsilon_{K}$ of $K \rightarrow$ $2 \pi$ decays. Therefore, it would be very useful to achieve an improved determination of $m_{s}$ with the BABAR and BELLE $\tau$ data samples. Taking $\left(m_{s}+m_{u, d}\right)\left(\mu^{2}\right)=(113 \pm 18) \mathrm{MeV}$, at $\mu=2 \mathrm{GeV}$, the Standard Model prediction of $\varepsilon_{K}^{\prime} / \varepsilon_{K}$ is found to be 70

$\operatorname{Re}\left(\varepsilon_{K}^{\prime} / \varepsilon_{K}\right)=\left(1.7 \pm 0.2_{-0.5}^{+0.8} \pm 0.5\right) \cdot 10^{-3}$,

where the largest error originates in the present uncertainty on $m_{s}$. This prediction agrees very well with the measured value $77,72,73,74$ $\operatorname{Re}\left(\varepsilon_{K}^{\prime} / \varepsilon_{K}\right)=(1.66 \pm 0.16) \cdot 10^{-3}$.

\section{NEUTRINO OSCILLATIONS}

The so-called "solar neutrino problem" has been a long-standing question, since the very first chlorine experiment at the Homestake mine [75]. The flux of solar $\nu_{e}$ neutrinos reaching the earth has been measured by several experiments [10] to be significantly below the standard solar model prediction [76]. The large Super-Kamiokande (SK) experiment [8], with its real time detection 


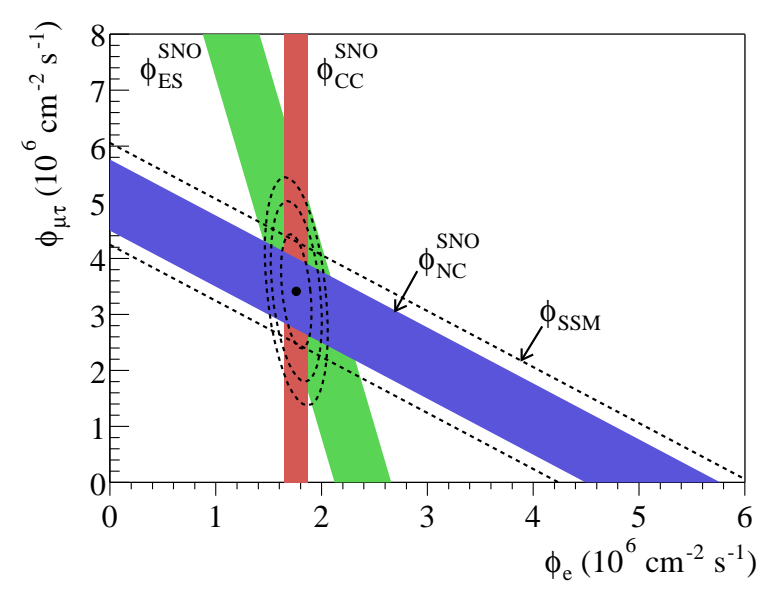

Figure 7. Measured flux of ${ }^{8} B$ solar neutrinos of $\nu_{\mu}$ or $\nu_{\tau}$ type $\left(\phi_{\mu, \tau}\right)$ versus the flux of $\nu_{e}\left(\phi_{e}\right)[7]$.

technique and direction sensitivity, has fully confirmed the deficit of solar electron neutrinos.

Very recently, the Sudbury Neutrino Observatory ( $\mathrm{SNO}$ ) has provided strong evidence that neutrinos do change flavour as they propagate from the core of the Sun [7], independently of solar model flux predictions. SNO is able to detect neutrinos through three different reactions:

$$
\begin{array}{ll}
\nu_{e}+d \rightarrow p+p+e^{-} & (\mathrm{CC}), \\
\nu_{x}+d \rightarrow p+n+\nu_{x} & (\mathrm{NC}), \\
\nu_{x}+e^{-} \rightarrow \nu_{x}+e^{-} & (\mathrm{ES}) .
\end{array}
$$

While the charged current (CC) reaction is only sensitive to $\nu_{e}$, the neutral current (NC) process has equal probability for all active neutrino flavours. The elastic scattering (ES) is also sensitive to $\nu_{\mu}$ and $\nu_{\tau}$, although the corresponding cross section is a factor 6.48 smaller than the $\nu_{e}$ one. The measured neutrino fluxes, shown in Fig. [7, demonstrate the existence of a non- $\nu_{e}$ component in the solar neutrino flux at the $5.3 \sigma$ level:

$\phi_{\mu \tau}=\left(3.41 \pm 0.45_{-0.45}^{+0.48}\right) \cdot 10^{6} \mathrm{~cm}^{-2} \mathrm{~s}^{-1}$.

The total neutrino flux measured with the NC process is consistent with the solar model prediction [76] (dotted line in Fig. 7).

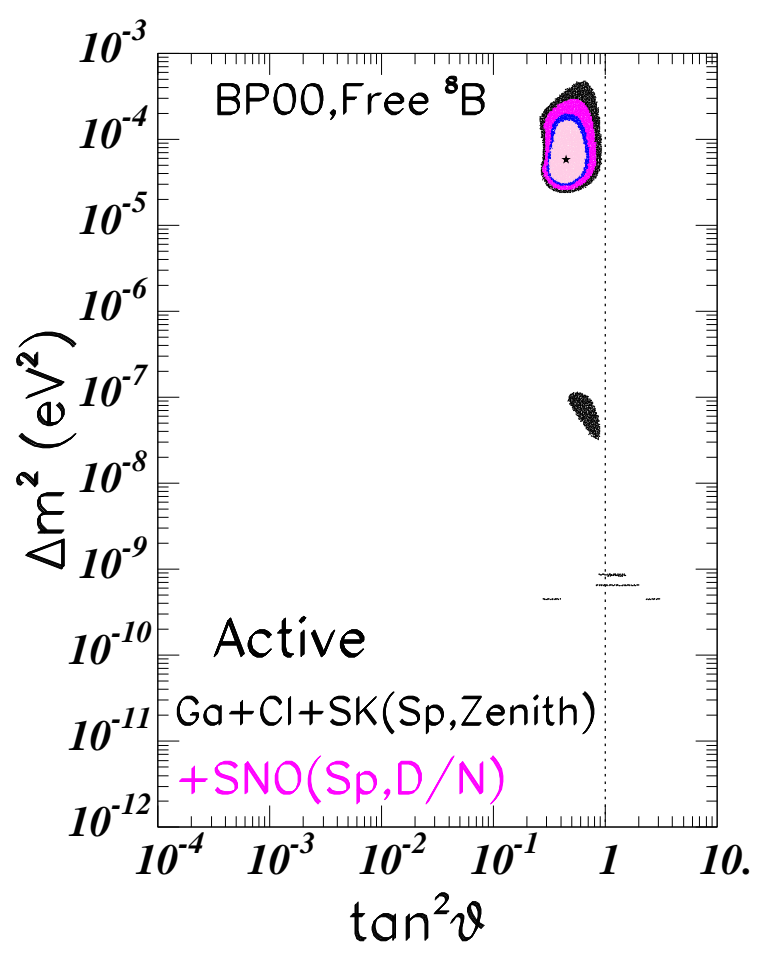

Figure 8. Allowed oscillation parameters (at 90, 95, 99 and $99.7 \%$ CL) from solar neutrinos 10, 77. The best fit point is marked with a star.

Another very important evidence of neutrino oscillations has been provided by the SK measurements of atmospheric neutrinos [9]. The known discrepancy between the experimental observations and the predicted ratio of muon to electron neutrinos has become much stronger with the high precision and large statistics of SK. The atmospheric anomaly has been identified to originate in a reduction of the $\nu_{\mu}$ flux, and the data strongly favours the $\nu_{\mu} \rightarrow \nu_{\tau}$ hypothesis. This result should be confirmed soon by K2K [78] and further studied at MINOS [79]. The direct detection of the produced $\nu_{\tau}$ is the main goal of the CERN to Gran Sasso neutrino program [80].

Figures 8 and 9 show the present information on neutrino oscillations, from solar and atmospheric experiments. A global analysis, combining the full set of solar, atmospheric and reac- 


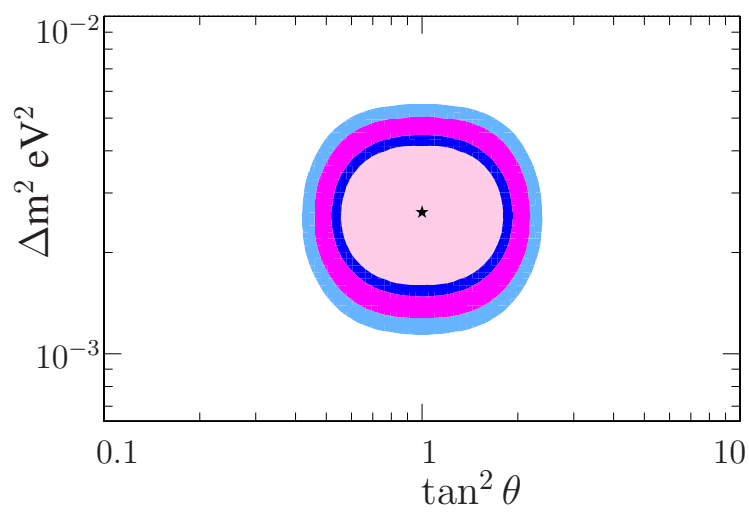

Figure 9. Allowed regions (at 90, 95, 99 and $99.7 \% \mathrm{CL}$ ) for $\nu_{\mu} \rightarrow \nu_{\tau}$ oscillations, from atmospheric neutrino data [10].

tor neutrino data, leads to the following preferred ranges for the oscillation parameters 10]:

$$
\begin{aligned}
& 2.4 \cdot 10^{-5}<\Delta m_{21}^{2} / \mathrm{eV}^{2}<2.4 \cdot 10^{-4}, \\
& 1.4 \cdot 10^{-3}<\Delta m_{32}^{2} / \mathrm{eV}^{2}<6.0 \cdot 10^{-3}, \\
& 0.27<\tan ^{2} \theta_{12}<0.77, \\
& 0.4<\tan ^{2} \theta_{23}<3.0, \\
& \sin ^{2} \theta_{13}<0.06,
\end{aligned}
$$

where $\Delta m_{i j}^{2} \equiv m_{i}^{2}-m_{j}^{2}$ are the mass squared differences between the neutrino mass eigenstates $\nu_{i, j}$ and $\theta_{i j}$ the corresponding mixing angles (in the standard three-flavour parameterization [11]). The angle $\theta_{13}$ is strongly constrained by the $\mathrm{CHOOZ}$ reactor experiment 81]. In the limit $\theta_{13}=0$, solar and atmospheric neutrino oscillations decouple because $\Delta m_{\odot}^{2} \ll \Delta m_{\text {atm }}^{2}$. Thus, $\Delta m_{21}^{2}, \theta_{12}$ and $\theta_{13}$ are constrained by solar data, while atmospheric experiments constrain $\Delta m_{32}^{2}$, $\theta_{23}$ and $\theta_{13}$.

\section{NEW PHYSICS}

The non-zero value of neutrino masses constitutes a clear indication of new physics beyond the Standard Model framework. The simplest modification would be to add the needed right-handed neutrino components to allow for
Dirac neutrino mass terms, generated through the electroweak spontaneous symmetry breaking mechanism. However, those $\nu_{i R}$ fields would be $S U(3)_{C} \otimes S U(2)_{L} \otimes U(1)_{Y}$ singlets and, therefore, would not have any Standard Model interaction (sterile neutrinos). If such objects do exist, it would seem natural to expect that they are able to communicate with the rest of the world through some still unknown dynamics. Moreover, the Standard Model gauge symmetry would allow for a right-handed Majorana neutrino mass term of arbitrary size, not related to the ordinary Higgs mechanism. Clearly, new physics is called for. If the Majorana masses are well above the electroweak symmetry breaking scale, the see-saw mechanism 82 leads to three light neutrinos at low energies.

We can make a more general analysis without any assumption about the existence of righthanded neutrinos or any other new particles at higher scales. Adopting an effective field theory language, one can write the most general $S U(3)_{C} \otimes S U(2)_{L} \otimes U(1)_{Y}$ invariant lagrangian, in terms of the known low-energy fields (left-handed neutrinos only):

$\mathcal{L}=\mathcal{L}_{\mathrm{SM}}+\sum_{d>4} \frac{c_{d}}{\Lambda^{d-4}} O_{d}$.

The lagrangian is ordered in terms of increasing dimensionality $d$; higher-dimension operators being suppressed by higher inverse powers of the new-physics scale $\Lambda$. The Standard Model is the unique answer with $d=4$. The first contributions from new physics appear at $d=5$ and have also a unique form 83]:

$\Delta \mathcal{L}=-\frac{c_{i j}}{\Lambda} \bar{L}_{i} \tilde{\phi} \tilde{\phi}^{t} L_{j}^{c}+$ h.c.,

where $\phi$ and $L_{i}$ are the scalar and $i$-flavoured lepton $S U(2)_{L}$ doublets, $\tilde{\phi} \equiv i \tau_{2} \phi^{*}$ and $L_{i}^{c} \equiv \mathcal{C} \bar{L}_{i}^{t}$. A similar operator with quark fields is forbidden by the gauge symmetry, due to the different hypercharges. After spontaneous symmetry breaking, $\left\langle\phi^{(0)}\right\rangle=v / \sqrt{2}$, the $d=5$ operator generates a Majorana mass term:

$\mathcal{L}_{M}=-\frac{1}{2} \bar{\nu}_{i L} M_{i j} \nu_{j L}^{c}+$ h.c., $\quad M_{i j}=\frac{c_{i j} v^{2}}{\Lambda}$. 
Thus, Majorana neutrino masses should be expected on general symmetry grounds. The relation (21) is nothing else that the see-saw mech$\operatorname{anism}\left(m_{\nu_{L}} \sim m^{2} / \Lambda\right)$. Taking $m_{\nu} \gtrsim 0.05 \mathrm{eV}$, as suggested by atmospheric neutrino data, one gets $\Lambda / c_{i j} \lesssim 10^{15} \mathrm{GeV}$, amazingly close to the expected scale of Gran Unification.

With non-zero neutrino masses, the leptonic charged current interactions,

$\mathcal{L}=\frac{g}{\sqrt{2}} W_{\mu}^{\dagger} \sum_{i j} \bar{\nu}_{i L} \gamma^{\mu} \mathbf{U}_{i j} l_{j L}+$ h.c.

involve a flavour mixing matrix U. Neglecting possible $\mathrm{CP}$-violating phases, the present data on neutrino oscillations implies the mixing structure:

$\mathbf{U} \approx\left[\begin{array}{ccc}\frac{1}{\sqrt{2}}(1+\lambda) & \frac{1}{\sqrt{2}}(1-\lambda) & \epsilon \\ -\frac{1}{2}(1-\lambda+\epsilon) & \frac{1}{2}(1+\lambda-\epsilon) & \frac{1}{\sqrt{2}} \\ \frac{1}{2}(1-\lambda-\epsilon) & -\frac{1}{2}(1+\lambda+\epsilon) & \frac{1}{\sqrt{2}}\end{array}\right]$,

with $\lambda \sim 0.2$ and $\epsilon<0.25$ [10]. Therefore, the mixing among leptons appears to be very different from the one in the quark sector.

An important question to be addressed in the future concerns the possibility of leptonic CP violation and its relevance for explaining the baryon asymmetry of our universe through a leptogenesis mechanism [84].

The smallness of the neutrino masses implies a strong suppression of neutrinoless lepton-flavourviolation processes. This suppression can be avoided in models with other sources of lepton flavour violation, not related to $m_{\nu_{i}}$ [85]. The present experimental limits on lepton-flavourviolating $\tau$ decays, at the $10^{-6}$ level [86,87, are already sensitive to new-physics scales of the order of a few $\mathrm{TeV}$ [88]. Further improvements at future experiments [89] would allow to explore interesting and totally unknown phenomena.

\section{SUMMARY}

Our knowledge of the lepton properties has been considerably improved during the last few years. Lepton universality has been tested to rather good accuracy, both in the charged and neutral current sectors. The Lorentz structure of the leptonic $l \rightarrow \nu_{l} l^{\prime} \bar{\nu}_{l^{\prime}}$ decays has been determined with good precision in the $\mu$ decay 11 and relevant constraints have been obtained for the $\tau$. An upper limit of $3.2 \%$ (90\% CL) has been already set on the probability of having a (wrong) decay from a right-handed $\tau$ [3, 3 ,

The quality of the hadronic $\tau$ decay data has made possible to perform quantitative QCD tests and determine the strong coupling constant very accurately, providing a nice experimental verification of asymptotic freedom. An improved value of the strange quark mass has been also obtained from Cabibbo-suppressed hadronic $\tau$ decays.

More recently, the precise determination of the $\mu$ anomalous magnetic moment has nearly reached the needed sensitivity to explore higherorder electroweak corrections. A further experimental improvement is expected soon. In order to perform a meaningful precision test of the electroweak theory, it is very important to achieve a better control of the QCD contributions.

The Standard Model provides a beautiful theoretical framework which is able to accommodate all our present knowledge on electroweak and strong interactions. In spite of this impressive phenomenological success, it leaves too many unanswered questions to be considered as a complete description of the fundamental forces. We do not understand yet why fermions are replicated in three (and only three) nearly identical copies. Why the pattern of masses and mixings is what it is? Are the masses the only difference among the three families? What is the origin of the flavour structure? Which dynamics is responsible for the observed $\mathrm{CP}$ violation?

The fermionic flavour is the main source of arbitrary free parameters in the Standard Model. The problem of fermion-mass generation is deeply related with the mechanism responsible for the electroweak spontaneous symmetry breaking. Thus, the origin of these parameters lies in the most obscure part of the Standard Model lagrangian: the scalar sector. Clearly, the dynamics of flavour appears to be "terra incognita" which deserves a careful investigation.

The first hints of new physics beyond the Standard Model have emerged recently, with convincing evidence of neutrino oscillations from both so- 
lar and atmospheric experiments. The existence of lepton flavour violation opens a very interesting window to unknown phenomena. New experiments will probe the Standard Model to a much deeper level of sensitivity and will explore the frontier of its possible extensions.

\section{ACKNOWLEDGEMENTS}

I would like to thank the organizers for hosting an enjoyable conference. I want to acknowledge useful discussions with V. Cirigliano, A. Czarnecki, M. Davier, M.C. González, F. Matorras, J. Portolés and P. Ruiz. This work has been supported in part by MCYT, Spain (Grant FPA2001-3031), by EU funds for regional development and by the EU, RTN EURIDICE (HPRN-CT2002-00311).

\section{REFERENCES}

1. A. Pich, The Standard Model of Particle Physics: Status \&6 Low-Energy Tests, hep$\mathrm{ph} / 0206011$.

2. M.W. Grünewald, Electroweak Physics, hepex/0210003.

3. A. Pich, Tau Physics, in Heavy Flavours II, eds. A.J. Buras and M. Lindner, Advanced Series on Directions in High Energy Physics 15 (World Scientific, Singapore, 1998), p. 453.

4. A. Pich, Tau Physics: Theoretical Perspective, in [5], p. 385.

5. R.J. Sobie and J.M. Roney (eds.), Proc. Sixth Workshop on Tau Lepton Physics (Victoria, 18-21 September 2000), Nucl. Phys. B (Proc. Suppl.) 98 (2001).

6. A. Stahl, Physics with Tau Leptons, Springer Tracts in Modern Physics 160 (Springer, Berlin, 2000).

7. SNO Collab., Phys. Rev. Lett. 89 (2002) 011301, 011302; G. Beier, these proceedings.

8. Super-Kamiokande Collab., Phys. Rev. Lett. 86 (2001) 5656; Phys. Lett. B 539 (2002) 179.

9. Super-Kamiokande Collab., Phys. Rev. Lett. 81 (1998) 1562, 85 (2000) 3999; hepex/0209036; C.K. Jung, these proceedings.

10. M.C. González-García, hep-ph/0210359; M.C. González-García and Y. Nir, hep- ph/0202058; J.J. Gómez-Cadenas, these proceedings.

11. Particle Data Group: Phys. Rev. D 66 (2002) 010001.

12. The LEP Collaborations ALEPH, DELPHI, L3, OPAL, the LEP Electroweak Working Group and the SLD Heavy Flavour Group: LEPEWWG/2002-01.

13. L. Kormos and F. Matorras, these proceedings.

14. A.J. Weinstein, these proceedings.

15. S. Eidelman, private communication.

16. P. Ruiz-Femenía and A. Pich, Phys. Rev. D 64 (2001) 053001; hep-ph/0210003; P. RuizFemenía, these proceedings.

17. F. Guerrero and A. Pich, Phys. Lett. B412 (1997) 382.

18. D. Gómez-Dumm, A. Pich and J. Portolés, Phys. Rev. D62 ( 2000$) 054014$.

19. A. Pich and J. Portolés, Phys. Rev. D 63 (2001) 093005; hep-ph/0209224.

20. J.J. Sanz-Cillero and A. Pich, hepph/0208199.

21. J. Gasser and H. Leutwyler, Nucl. Phys. B250 (1985) 465, 517, 539.

22. G. Ecker, Prog. Part. Nucl. Phys. 35 (1995) 1; A. Pich, Rep. Prog. Phys. 58 (1995) 563.

23. G. Ecker et al, Nucl. Phys. B321 (1989) 311; Phys. Lett. 233B (1989) 425.

24. G. 't Hooft, Nucl. Phys. B72 (1974) 461;

E. Witten, Nucl. Phys. B160 (1979) 57.

25. A. Pich, Colourless Mesons in a Polychromatic World, hep-ph/0205030.

26. ALEPH Collab., Z. Phys. C76 (1997) 15.

27. CLEO Collab., Phys. Rev. D61 (2000) 112002.

28. G. Ecker and R. Unterdorfer, Eur. Phys. J. C 24 (2002) 535.

29. CLEO Collab., Phys. Rev. D61 (2000) 052004, 012002; E.I. Shibata, these proceedings.

30. OPAL Collab., Z. Phys. C75 (1997) 593.

31. D. Gómez-Dumm, A. Pich and J. Portolés, in progress.

32. V.H. Hughes and T. Kinoshita, Rev. Mod. Phys. 71 (1999) S133.

33. T. Kinoshita and M. Nio, hep-ph/0210322.

34. A. Czarnecki and W.J. Marciano, Phys. Rev. 
D 64 (2001) 013014; A. Czarnecki, these proceedings.

35. R.S. Van Dyck Jr., P.B. Schwinberg and H.G. Dehmelt, Phys. Rev. Lett. 59 (1987) 26.

36. Muon $(g-2)$ Collab., Phys. Rev. Lett. 86 (2001) 2227, 89 (2002) 101804; L. Roberts, these proceedings.

37. A. Czarnecki et al., Phys. Rev. Lett. 76 (1996) 3267; Phys. Rev. D 52 (1995) 2619; T.V. Kukhto et al., Nucl. Phys. B 371 (1992) 567; S. Peris et al, Phys. Lett. B 355 (1995) 523; M. Knecht et al., hep-ph/0205102.

38. M. Davier , S. Eidelman, A. Höcker and Z. Zhang, hep-ph/0208177; these proceedings.

39. B. Krause, Phys. Lett. B 390 (1997) 392.

40. M. Knecht and A. Nyffeler, Phys. Rev. D 65 (2002) 073034; M. Knecht et al., Phys. Rev. Lett. 88 (2002) 071802; I. Blokland et al., Phys. Rev. Lett. 82 (2002) 071803; J. Bijnens et al., Nucl. Phys. B 626 (2002) 410. M. Hayakawa and T. Kinoshita, hep-ph/0112102; M.J. Ramsey-Musolf and M. Wise, Phys. Rev. Lett. 89 (2002) 041601.

41. M. Hayakawa and T. Kinoshita, Phys. Rev. D 57 (1998) 465, Err: 66 (2002) 019902; J. Bijnens et al., Nucl. Phys. B 474 (1996) 379; Phys. Rev. Lett. 75 (1995) 1447, 3781.

42. K. Hagiwara et al., hep-ph/0209187; F. Jegerlehner, hep-ph/0104304; J.F. de Trocóniz and F.J. Ynduráin, Phys. Rev. D 65 (2002) 093001, 093002; S. Narison, Phys. Lett. B 513 (2001) 53; 526 (2002) 414; M. Davier and A. Höcker, Phys. Lett. B (1998) 435, 427; R. Alemany et al., Eur. Phys. J. C 2 (1998) 123;

43. V. Cirigliano, G. Ecker and H. Neufeld, Phys. Lett. B 513 (2001) 361; JHEP 08 (2002) 002.

44. G. Rodrigo and G. Venanzoni, these proceedings.

45. E. Braaten, Phys. Rev. Lett. 60 (1988) 1606; Phys. Rev. D39 (1989) 1458.

46. S. Narison and A. Pich, Phys. Lett. B211 (1988) 183.

47. E. Braaten, S. Narison and A. Pich, Nucl. Phys. B373 (1992) 581.

48. F. Le Diberder and A. Pich, Phys. Lett. B286 (1992) 147.
49. A. Pich, Nucl. Phys. B (Proc. Suppl.) 39B,C (1995) 326.

50. W.J. Marciano and A. Sirlin, Phys. Rev. Lett. 61 (1988) 1815.

51. E. Braaten and C.S. Li, Phys. Rev. D42 (1990) 3888.

52. F. Le Diberder and A. Pich, Phys. Lett. B289 (1992) 165.

53. ALEPH Collab., Eur. Phys. J. C4 (1998) 409; Phys. Lett. B307 (1993) 209.

54. CLEO Collab., Phys. Lett. B356 (1995) 580.

55. OPAL Collab., Eur. Phys. J. C7 (1999) 571.

56. G. Rodrigo, A. Pich and A. Santamaria, Phys. Lett. B424 (1998) 367.

57. ALEPH Collab., Eur. Phys. J. C11 (1999) 599, C10 (1999) 1.

58. A. Stahl and W.F. Mader, private communication.

59. A. Pich and J. Prades, JHEP 10 (1999) 004; 06 (1998) 013.

60. S. Chen et al., Eur. Phys. J. C 22 (2001) 31.

61. K.G. Chetyrkin and A. Kwiatkowski, Z. Phys. C59 (1993) 525; hep-ph/9805232.

62. K.G. Chetyrkin, Phys. Lett. B390 (1997) 309; K.G. Chetyrkin and J.H. Kühn, Phys. Lett. B406 (1997) 102.

63. K. Maltman, Phys. Rev. D58 (1998) 093015; these proceedings.

64. K.G. Chetyrkin et al, Nucl. Phys. B533 (1998) 473; J.G. Körner et al, Eur. Phys. J. C 20 (2001) 259.

65. J. Kambor and K. Maltman, Phys. Rev. D62 (2000) 093023.

66. V. Lubicz, Nucl. Phys. B (Proc. Suppl.) 94 (2001) 116; R. Gupta and K. Maltman, Int. J. Mod. Phys. A 16S1B (2001) 591; H. Wittig, hep-lat/0210025.

67. M. Jamin, J.A. Oller and A. Pich, Eur. Phys. J. C 24 (2002) 237.

68. K. Maltman and J. Kambor, Phys. Rev. D 65 (2002) 074013.

69. M. Jamin, J.A. Oller and A. Pich, Nucl. Phys. B 622 (2002) 279, 587 (2000) 331.

70. E. Pallante, A. Pich and I. Scimemi, Nucl. Phys. B 617 (2001) 441.

71. NA48 Collab., Phys. Lett. B 544 (2002) 97, Eur. Phys. J. C 22 (2001) 231; Phys. Lett. B 465 (1999) 335. 
72. NA31 Collab., Phys. Lett. B 317 (1993) 233.

73. KTeV Collab., hep-ex/0208007; Phys. Rev. Lett. 83 (1999) 22.

74. E731 Collab., Phys. Rev. Lett. 70 (1993) 1203.

75. R. Davis et al., Phys. Rev. Lett. 20 (1968) 1205.

76. J. Bahcall et al., JHEP 04 (2002) 007.

77. J.N. Bahcall, M.C. González-García and C. Peña-Garay, JHEP 07 (2002) 054.

78. C. Walter, these proceedings.

79. M. Diwan, these proceedings.

80. D. Duchesneau and V. Palladino, these proceedings.

81. CHOOZ Collab., Phys. Lett. B 466 (1999) 415.

82. M. Gell-Mann, P. Ramond and R. Slansky, Complex Spinors and Unified Theories, in Supergravity, eds. P. van Nieuwenhuizen and D.Z. Freedman (North Holland, Stony Brook 1979) 315; T. Yanagida, in Proc. Workshop on Unified Theory and Baryon Number in the Universe, eds. O. Sawada and A. Sugamoto (KEK, 1979) 95.

83. S. Weinberg, Phys. Rev. Lett. 43 (1979) 1566.

84. M. Fukugita and T. Yanagida, Phys. Lett. B 174 (1986) 45; W. Buchmüller and M. Plümacher, Int. J. Mod. Phys. A 15 (2000) 5047.

85. E. Ma, these proceedings.

86. CLEO Collab., Phys. Rev. D 66 (2002) 071101, 61 (2000) 071101, 57 (1998) 5903, 55 (1997) 3919; Phys. Rev. Lett. 79 (1997) 1221.

87. K. Inami et al., these proceedings.

88. D. Black et al., Phys. Rev. D 66 (2002) 053002 .

89. R. Santinelli, these proceedings. 